\title{
Sistemas cristalinos: nomenclatura e convenções
}

\author{
Crystal systems: nOMEnCLature AND CONVENTIONS \\ Daniel Atencio ${ }^{1,2}$ e Andrezza de Almeida Azzi ${ }^{1,3}$ \\ 1- InSTITUTO DE GEOCIÊNCIAS - UNIVERSIDADE DE SÃO PAULO, RUA DO LAGO, 562 - 05508-080 - SÃO PAULO - SP - BRASIL. \\ 2-datencio@usp.br \\ 3- andrezzazzi@gmail.com
}

ABSTRACT: Crystal systems are presented in crystallography introductory books according to the set of symmetry elements and with respect to the axial orientation. However, what is fundamental for the crystal system definition is only the symmetry elements set and not the axial orientation. Some of the names applied to crystal systems are actually connected to the symmetry elements: trigonal, tetragonal, and hexagonal. The other systems have names related to the axial orientation: cubic, orthorhombic, monoclinic, and triclinic. It seems logical to think that the triclinic crystals are those that have all angles different from $90^{\circ}$ and that the monoclinic crystals are those that present only one angle different of $90^{\circ}$, which is not always the case. "Diclinic" crystals, with one angle equal to $90^{\circ}$ and two different, also exist, and are grouped with the triclinic ones, because they present the same crystal classes. The conventions for nomenclature of crystal systems could be more logical. One option would be to use names for all systems, which relate to the symmetry elements. The cubic system would be called tetra-trigonal system, the orthorhombic system would be called tri-digonal system, the monoclinic system would be called digonal system and the triclinic system could be renamed monogonal system. These four names are logical and technically correct, unlike those that are officially used.

\section{Manuscrito:}

Recebido: 23/10/2017

Corrigido: $13 / 11 / 2017$

Aceito: $14 / 11 / 2017$

Citation: Atencio D., Azzi A.A. 2017. Sistemas cristalinos: nomenclatura e convenções.Terræ Didatica, 13(3):279285. <http://www.ige.unicamp.br/terraedidatica/ $>$.

Keywords: crystal systems, axial orientation, diclinic system, crystallographic nomenclature, symmetry elements.

\section{Introdução}

A Cristalografia é uma ciência cheia de regras (convenções internacionais) que foram feitas para que pessoas do mundo todo usem as mesmas orientações cristalográficas e nomenclaturas, de modo a que possam se entender. Se cada um usasse regras diferentes para orientar os cristais e classificá-los, por exemplo, quanto ao grupo pontual, classe cristalina e sistema cristalino, a Cristalografia iria se tornar muito complexa. Estas questões não são, em geral, muito claras nos livros introdutórios de Cristalografia, como naqueles voltados para sua aplicação na Mineralogia, como Bloss (1971), Chvátal (2007) e Klein \& Dutrow (2012). Por outro lado, as convenções atuais, padronizadas pela IUCr (International Union of Crystallography), são algumas vezes impróprias. Por exemplo, ao denominarem triclínico um sistema no qual não obrigatoriamente todos os ângulos da cela unitária são diferentes de $90^{\circ}$, ou ao denominarem monoclínicos cristais que podem ter ângulos todos iguais a $90^{\circ}$.

\section{Fundamentação}

Algo que atrai muito a atenção nos cristais é a presença de faces planas. Estas faces podem se repetir algumas vezes, apresentando-se rotacionadas em relação umas as outras (são faces idênticas e podem ser vistas após uma rotação do cristal), ou podem estar refletidas (uma é a imagem espelhada da outra), ou invertidas (uma é o inverso da outra). Esta repetição é conhecida como simetria. Para descrever a simetria, podem ser utilizados alguns elementos fictícios, chamados de elementos de simetria, que nos permitem imaginar como as faces dos cristais se "reproduzem". Um deles é o eixo de simetria próprio, uma linha imaginária, ao redor da qual aparecem faces repetidas 2, 3, 4 ou 6 vezes. Outro elemento de simetria é o plano de simetria, que funciona como um espelho e faz com que as faces apareçam aos pares, sendo uma a imagem especular da outra. Um terceiro elemento é o centro de simetria, que, quando existente, fica no centro geométrico do cristal e faz com que para todas as faces apareça o seu 
inverso do outro lado do cristal. O último elemento de simetria é o eixo de simetria impróprio, que a cada operação de rotação, inverte a face do cristal. Estes elementos estão representados na Figura 1.

Cada cristal apresenta uma associação de elementos de simetria. Por exemplo, imaginemos um cristal com a forma de um cubo perfeito. Nele vamos encontrar 3 eixos de simetria próprios de ordem 4, 4 eixos de simetria impróprios de ordem 3, 6 eixos de

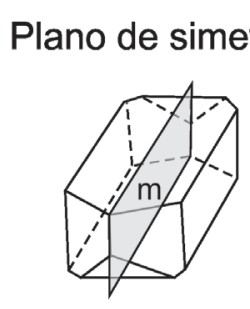

\section{Centro de simetria (i)}

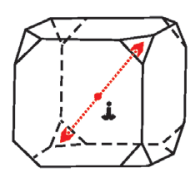

\section{Eixos de simetria próprios}
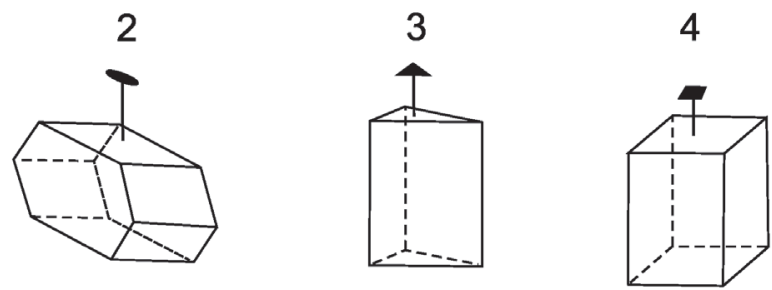

\begin{tabular}{|c|c|c|c|c|c|}
\hline Denominação do Eixo & Frequência, $\mathbf{n}$ & $\begin{array}{c}\text { Ângulo de rotação } \alpha, \\
\text { rad. }\end{array}$ & Índice & Marca gráfica & $\begin{array}{c}\text { Número máximo no } \\
\text { cristal }\end{array}$ \\
\hline Unitário & 1 & 360 & 1 & & \\
Binário & 2 & 180 & 2 & & 6 \\
Ternário & 3 & 120 & 3 & 4 & 4 \\
Quaternário & 4 & 90 & 4 & & 3 \\
Senário & 6 & 60 & 6 & & 1 \\
\hline
\end{tabular}

\section{Eixos de simetria impróprios}

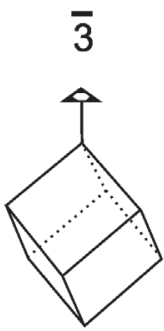

$\overline{4}$

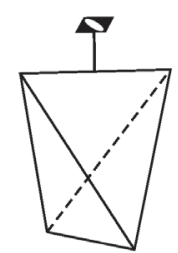

\begin{tabular}{|c|c|c|c|c|c|}
\hline Denominação do Eixo & Frequência, $\mathbf{n}$ & $\begin{array}{c}\text { Ângulo de rotação } \alpha \\
\text { rad. }\end{array}$ & Índice & Marca gráfica & $\begin{array}{c}\text { Número máximo no } \\
\text { cristal }\end{array}$ \\
\hline Unitário inverso & 1 & 360 & 1 & & \\
Binário inverso & 2 & 180 & $\overline{2}$ & & \\
Ternário inverso & 3 & 120 & $\overline{3}$ & $\Delta$ & 4 \\
Quaternário inverso & 4 & 90 & $\overline{4}$ & $\square$ & 3 \\
Senário inverso & 6 & 60 & $\overline{6}$ & $\Delta$ & 1 \\
\hline
\end{tabular}

Figura 1. Elementos de simetria

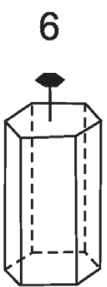

$\overline{6}$

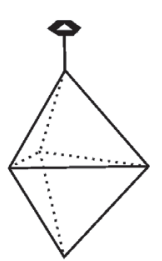

simetria próprios de ordem 2, e 9 planos de simetria. Verificamos também que este cristal apresenta centro de simetria. Esta, então, é a associação de elementos de simetria (= grau de simetria) do cristal cuja forma é denominada de cubo (Fig. 2).

Existem apenas 32 possibilidades de associações de elementos de simetria, também chamadas de grupos pontuais (grupo de elementos de simetria ao redor de um ponto, localizado no centro geométrico do cristal). Diz-se que todos os cristais que apresentam um mesmo grupo pontual pertencem a uma mesma classe cristalina e as classes cristalinas são agrupadas em sistemas cristalinos. Por exemplo, as classes cristalinas que apresentam um eixo de simetria de ordem 6 (próprio ou impróprio) pertencem ao sistema cristalino hexagonal. Todas estas informações estão resumidas na Tabela 1.

Na Tabela 1, podemos notar algo que parece estranho: há um sistema monoclínico e um triclínico, mas não há um diclínico... Vamos conversar sobre isso daqui a pouco.

Com a finalidade de dar "nomes" às faces e direções dos cristais, eles são espacialmente orientados. Para tanto, utiliza-se outro conjunto de eixos imaginários, os eixos cristalográficos. Estes eixos são em número de 3 ou 4, dependendo do sistema cristalino. Eles se cruzam formando então determinados ângulos interaxiais. $\mathrm{O}$ conjunto entre eixos cristalográficos e seus respectivos ângulos interaxiais é chamado de cruz axial (Fig. 3).

Mas por que os cristais apresentam essa simetria externa? Por 


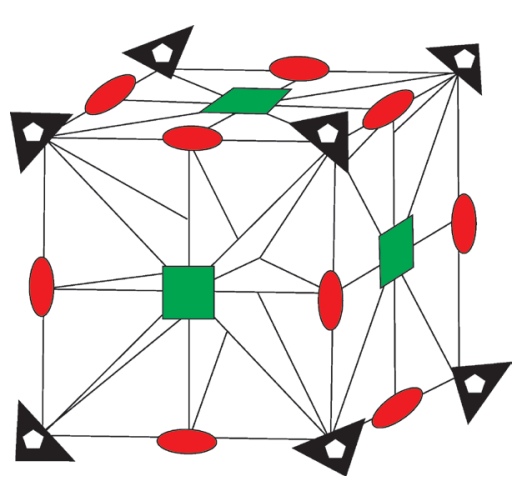

Figura 2. Elementos de simetria de um cubo
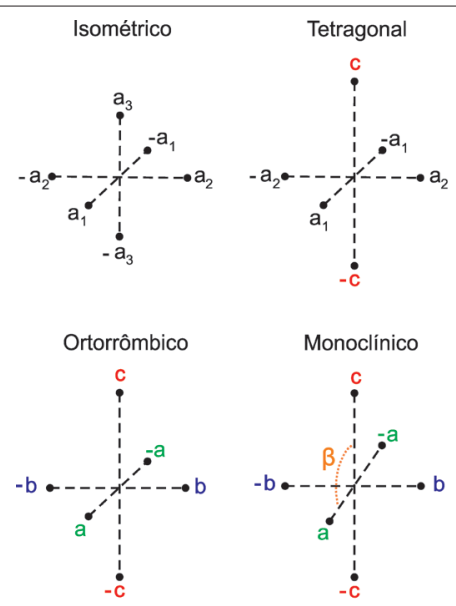

Figura 3. Cruzes axiais
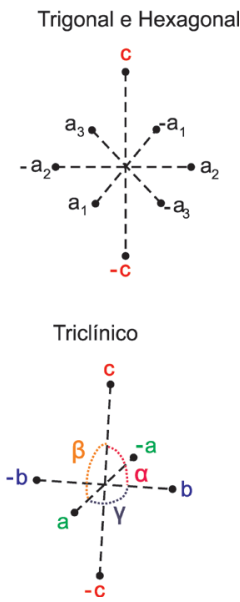

Tabela 1. Os sistemas cristalinos e suas classes

\begin{tabular}{|c|c|c|c|}
\hline Sistema Cristalino & Classe Cristalina & Grau de Simetria & Nome da Classe \\
\hline \multirow{2}{*}{ Triclínico } & 1 & Sem simetria & Pedial \\
\hline & $\overline{1}$ & $\mathrm{i}$ & Pinacoidal \\
\hline \multirow{3}{*}{ Monoclínico } & 2 & 1E2 & Esfenoédrica \\
\hline & $\mathrm{m}$ & $1 \mathrm{~m}$ & Domática \\
\hline & $2 / \mathrm{m}$ & $1 \mathrm{E} 2,1 \mathrm{~m}, \mathrm{i}$ & Prismática \\
\hline \multirow{3}{*}{ Ortorrômbico } & 222 & $3 \mathrm{E} 2$ & Biesfenoédrica rômbica \\
\hline & $\mathrm{mm} 2$ & $1 \mathrm{E} 2,2 \mathrm{~m}$ & Piramidal rômbica \\
\hline & $2 / \mathrm{m} 2 / \mathrm{m} 2 / \mathrm{m}$ & $3 \mathrm{E} 2,3 \mathrm{~m}, \mathrm{i}$ & Bipiramidal rômbica \\
\hline \multirow{7}{*}{ Tetragonal } & 4 & $1 \mathrm{E} 4$ & Piramidal tetragonal \\
\hline & $\overline{4}$ & $1 \mathrm{E} \overline{4}$ & Biesfenoédrica tetragonal \\
\hline & $4 / \mathrm{m}$ & $1 \mathrm{E} 4,1 \mathrm{~m}, \mathrm{i}$ & Bipiramidal tetragonal \\
\hline & $\overline{4} 2 \mathrm{~m}$ & $1 \mathrm{E} \overline{4}, 2 \mathrm{E} 2,2 \mathrm{~m}$ & Escalenoédrica tetragonal \\
\hline & 422 & $1 \mathrm{E} 4,4 \mathrm{E} 2$ & Trapezoédrica tetragonal \\
\hline & $4 \mathrm{~mm}$ & $1 \mathrm{E} 4,4 \mathrm{~m}$ & Piramidal ditetragonal \\
\hline & $4 / \mathrm{m} 2 / \mathrm{m} \mathrm{2} / \mathrm{m}$ & 1E4, 4E2, 5m, i & Bipiramidal ditetragonal \\
\hline \multirow{5}{*}{ Trigonal } & 3 & $1 \mathrm{E} 3$ & Piramidal trigonal \\
\hline & $\overline{3}$ & $1 \mathrm{E} \overline{3}$ & Romboédrica \\
\hline & $\overline{3} 2 / \mathrm{m}$ & $1 \mathrm{E} \overline{3}, 3 \mathrm{E} 2,3 \mathrm{~m}, \mathrm{i}$ & Escalenoédrica hexagonal \\
\hline & 32 & 1E3, 3E2 & Trapezoédrica trigonal \\
\hline & $3 \mathrm{~m}$ & $1 \mathrm{E} 3,3 \mathrm{~m}$ & Piramidal ditrigonal \\
\hline \multirow{7}{*}{ Hexagonal } & 6 & $1 \mathrm{E} 6$ & Piramidal hexagonal \\
\hline & $\overline{6}$ & $1 \mathrm{E} \overline{6}$ & Bipiramidal trigonal \\
\hline & $6 / \mathrm{m}$ & $1 \mathrm{E} 6,1 \mathrm{~m}, \mathrm{i}$ & Bipiramidal hexagonal \\
\hline & $\overline{6} \mathrm{~m} 2$ & $1 \mathrm{E} \overline{6}, 3 \mathrm{E} 2,3 \mathrm{~m}$ & Bipiramidal ditrigonal \\
\hline & 622 & 1E6, 6E2 & Trapezoédrica hexagonal \\
\hline & $6 \mathrm{~mm}$ & $1 \mathrm{E} 6,6 \mathrm{~m}$ & Piramidal dihexagonal \\
\hline & $6 / \mathrm{m} \mathrm{2} / \mathrm{m} \mathrm{2} / \mathrm{m}$ & $1 \mathrm{E} 6,6 \mathrm{E} 2,7 \mathrm{~m}, \mathrm{i}$ & Bipiramidal dihexagonal \\
\hline \multirow{5}{*}{$\begin{array}{l}\text { Isométrico } \\
\text { (Cúbico) }\end{array}$} & 23 & 4E3, 3E2 & Tetartoédrica \\
\hline & $2 / \mathrm{m} \overline{3}$ & $4 \mathrm{E} \overline{3}, 3 \mathrm{E} 2,3 \mathrm{~m}, \mathrm{i}$ & Diploédrica \\
\hline & $\overline{4} 3 \mathrm{~m}$ & $4 \mathrm{E} 3,3 \mathrm{E} \overline{4}, 6 \mathrm{~m}$ & Hexatetraédrica \\
\hline & 432 & 4E3, 3E4, 6E2 & Giroédrica \\
\hline & $4 / \mathrm{m} \overline{3} 2 / \mathrm{m}$ & $4 \mathrm{E} \overline{3}, 3 \mathrm{E} 4,6 \mathrm{E} 2,9 \mathrm{~m}, \mathrm{i}$ & Hexaoctaédrica \\
\hline
\end{tabular}


causa de uma simetria interna! Os átomos acham-se arranjados dentro de um cristal e se observarmos sua distribuição no mesmo, veremos que eles estão ordenadamente distribuídos, formando como que "tijolos" que constroem uma parede. Os tijolos, que repetidos formam toda uma estrutura cristalina, são chamados de cela unitária. As dimensões da cela unitária (ou seja, as arestas do tijolo) são chamadas de parâmetros da cela unitária. Como as arestas da cela coincidem com os eixos cristalográficos, as letras utilizadas para definir os eixos cristalográficos, que são adimensionais, são as mesmas aplicadas para as dimensões da cela unitária. Isto pode, eventualmente, causar alguma confusão.
Os sistemas cristalinos são apresentados sob dois aspectos nos livros introdutórios de cristalografia: (a) com relação ao conjunto de elementos de simetria, e (b) com relação à cruz axial.

\section{Elementos de simetria}

Quanto ao primeiro aspecto, os elementos de simetria, têm-se as informações da Tabela 2 .

\section{Cruz axial}

Quanto ao segundo aspecto, a cruz axial, as informações são normalmente apresentadas como na Tabela 3:

Tabela 2: Agrupamento de classes cristalinas nos diferentes sistemas cristalinos.

\begin{tabular}{|c|c|c|}
\hline SISTEMA CRISTALINO & CONDIÇÕES & $\mathrm{N}^{\circ}$ CLASSES \\
\hline TRICLÍNICO & $\begin{array}{l}\text { Nenhum elemento de simetria presente ou apenas um } \\
\text { centro de simetria }\end{array}$ & 2 \\
\hline MONOCLÍNICO & $\begin{array}{l}\text { Apenas } 1 \text { eixo de ordem } 2 \text { e/ou um plano de simetria. } \\
\text { Como um plano de simetria equivale a um eixo impróprio } \\
\text { de ordem } 2 \text {, pode-se também dizer que os cristais do sistema } \\
\text { monoclínico apresentam apenas um eixo } 2 \text { ou } \overline{2}\end{array}$ & 3 \\
\hline ORTORRÔMBICO & $\begin{array}{l}\text { Três eixos 2, ou três eixos } 2 \text { com planos de simetria per- } \\
\text { pendiculares, ou } 2 \text { planos de simetria e um eixo } 2\end{array}$ & 3 \\
\hline TRIGONAL & $\begin{array}{l}\text { Apenas } 1 \text { eixo de ordem } 3 \text { ou } \overline{3} \text { acompanhado ou não por } \\
\text { eixos de ordem } 2 \text { e planos de simetria }\end{array}$ & 5 \\
\hline HEXAGONAL & $\begin{array}{l}\text { Apenas } 1 \text { eixo } 6 \text { ou } \overline{6} \text { acompanhado ou não por eixos } 2 \\
\text { e planos de simetria }\end{array}$ & 7 \\
\hline TETRAGONAL & $\begin{array}{l}\text { Apenas } 1 \text { eixo } 4 \text { ou } \overline{4} \text { acompanhado ou não por eixos de } \\
\text { ordem } 2 \text { e planos de simetria }\end{array}$ & 7 \\
\hline ISOMÉTRICO & $\begin{array}{l}4 \text { eixos de ordem } 3 \text { ou } \overline{3} \text {, acompanhados por } 3 \text { eixos } \\
\text { de ordem } 2,4 \text { ou } \overline{4} \text {, com ou sem outros eixos de ordem } 2 \text { e } \\
\text { planos de simetria }\end{array}$ & 5 \\
\hline
\end{tabular}

Tabela 3: Os sistemas cristalinos e as características das cruzes axiais

\begin{tabular}{|c|c|c|}
\hline SISTEMAS & $\begin{array}{c}\text { CONSTANTES } \\
\text { LINEARES }\end{array}$ & $\begin{array}{l}\text { CONSTANTES } \\
\text { ANGULARES }\end{array}$ \\
\hline TRICLÍNICO & $a^{1} b^{1} c$ & $a^{1} b^{1} g^{1} 90^{\circ}$ \\
\hline MONOCLÍNICO & $a^{1} b^{1} c$ & $a=g=90^{\circ}{ }^{1} b$ \\
\hline ORTORRÔMBICO & $a^{1} b^{1} c$ & $a=b=g=90^{\circ}$ \\
\hline TRIGONAL & $a_{1}=a_{2}=a_{3}{ }^{l} c$ & $a=b=90^{\circ}{ }^{1} \mathrm{~g}=120^{\circ}$ \\
\hline HEXAGONAL & $a_{1}=a_{2}=a_{3}{ }^{l} c$ & $a=b=90^{\circ}{ }^{1} \mathrm{~g}=120^{\circ}$ \\
\hline TETRAGONAL & $\begin{aligned} a & =b^{1} c \\
a_{1} & =a_{2}{ }^{1} c\end{aligned}$ & $a=b=g=90^{\circ}$ \\
\hline $\begin{array}{c}\text { ISOMÉTRICO } \\
\text { (CÚBICO) }\end{array}$ & $\begin{array}{c}a=b=c \\
a_{1}=a_{2}=a_{3}\end{array}$ & $a=b=g=90^{\circ}$ \\
\hline
\end{tabular}




\section{Aplicação}

Vamos então tentar aplicar o conteúdo das tabelas 1, 2 e 3 para alguns exemplos de minerais cujas informações a respeito de parâmetros de cela unitária e classe cristalina estão apresentadas a seguir nas tabelas 4, 5 e 6. Excluiremos da discussão os compostos cristalinos dos sistemas trigonal e hexagonal para dar enfoque aos sistemas cristalinos "mal padronizados".

Os minerais da Tabela 4 apresentam os parâmetros de cela unitária $a, b$ e $c$ diferentes entre si, dois ângulos diferentes de $90^{\circ}$ e um igual a $90^{\circ}$. Pertenceriam, de acordo com sua cruz axial, ao sistema diclínico, nome que não está nas tabelas anteriores, mas que já foi utilizado na literatura cristalográfica (ver discussão em Roger, 1938, e Pertlik, 2006). No sistema monoclínico, só um dos ângulos é diferente de $90^{\circ}$ e no triclínico, os três ângulos são diferentes de $90^{\circ}$. Quando dois ângulos são diferentes de $90^{\circ}$, o sistema deveria ser diclínico, mas estes minerais se enquadram formalmente no sistema triclínico, pois todos pertencem às classes $1 \mathrm{e} \overline{\mathbf{1}}$. O que define o sistema são as classes cristalinas e não a cruz axial.

Os minerais da Tabela 5 apresentam os parâmetros de cela unitária $a, b$ e $c$ diferentes entre si, dois ângulos iguais a $90^{\circ} \mathrm{e} \mathrm{um} \mathrm{diferente} \mathrm{de} 90^{\circ}$. No sistema monoclínico, só um dos ângulos é diferente de $90^{\circ}$ e no triclínico, os três ângulos são diferentes de $90^{\circ}$. Pertenceriam, de acordo com sua cruz axial, ao sistema monoclínico, mas se enquadram formalmente no sistema triclínico, pois todos pertencem às classes $1 \mathrm{e} \overline{1}$.

Os minerais da Tabela 6 apresentam os parâmetros de cela unitária $a, b$ e $c$ diferentes entre si e os três ângulos iguais a $90^{\circ}$. No sistema ortorrômbico todos os ângulos são iguais a $90^{\circ} \mathrm{e}$, no sistema

Tabela 4. Parâmetros de cela unitária e classe cristalina de alguns minerais triclínicos pseudo-diclínicos.

\begin{tabular}{|c|c|c|c|c|c|c|c|}
\hline mineral & $a(\AA)$ & $b(\AA)$ & $c(\AA)$ & $\alpha\left(^{\circ}\right)$ & $\beta\left(^{\circ}\right)$ & $\gamma\left({ }^{\circ}\right)$ & classe \\
\hline calcioaravaipaíta & 7,72 & 7,51 & 12,2 & 98,86 & 96,91 & 90 & $\overline{1}$ \\
\hline carlhintzeíta & 9,485 & 6,983 & 9,305 & 91,14 & 104,85 & 90 & 1 ou $\overline{1}$ \\
\hline cilindrita & $\begin{array}{l}11,733 \\
11,709 \\
\end{array}$ & $\begin{array}{l}5,790 \\
3,670 \\
\end{array}$ & $\begin{array}{l}5,810 \\
6,320 \\
\end{array}$ & $\begin{array}{l}90 \\
90 \\
\end{array}$ & $\begin{array}{l}92,38 \\
92,58 \\
\end{array}$ & $\begin{array}{l}93,87 \\
90,85\end{array}$ & 1 \\
\hline clairita & 9,36 & 9,15 & 52,61 & 88,15 & 90 & 118,36 & 1 ou $\overline{1}$ \\
\hline cookeíta-1a & 5,14 & 8,90 & 14,15 & 90,5 & 96,2 & 90 & $\overline{1}$ \\
\hline götzenita & 9,667 & 5,731 & 7,334 & 90 & 101,05 & 101,31 & $\overline{1}$ \\
\hline innelita & 14,76 & 7,14 & 5,38 & 90 & 95 & 99 & 1 \\
\hline lengenbachita & 36,892 & 5,842 & 5,847 & 90 & 92 & 91,01 & 1 ou $\overline{1}$ \\
\hline lomonosovita & 5,49 & 7,11 & 14,5 & 101 & 96 & 90 & $\overline{1}$ \\
\hline mcnearita & 13,5 & 14,1 & 6,95 & 90 & 92 & 119 & 1 ou $\overline{1}$ \\
\hline metavanuralita & 10,46 & 8,44 & 10,43 & 75,88 & 102,83 & 90 & 1 ou $\overline{1}$ \\
\hline minnesotaíta & 5,623 & 9,419 & 9,624 & 85,21 & 95,64 & 90 & $\overline{1}$ \\
\hline tantita & 3,8 & 3,78 & 35,74 & 90,91 & 90,19 & 90 & 1 ou $\overline{1}$ \\
\hline
\end{tabular}

Tabela 5. Parâmetros de cela unitária e classe cristalina de alguns minerais triclínicos pseudo-monoclínicos.

\begin{tabular}{c|c|c|c|c|c|c|c}
\hline mineral & $a(\AA)$ & $b(\AA)$ & $c(\AA)$ & $\alpha\left(^{\circ}\right)$ & $\beta\left(^{\circ}\right)$ & $\gamma\left(^{\circ}\right)$ & classe \\
\hline astrofilita & 11,76 & 5,36 & 21,08 & 90 & 94 & 90 & $\overline{1}$ \\
\hline baileycloro & 5,346 & 9,257 & 14,40 & 90 & 97,12 & 90 & 1 ou $\overline{1}-$ \\
\hline foshagita & 10,32 & 7,36 & 14,07 & 90 & 106,4 & 90 & $\overline{1}$ \\
\hline franckeíta & 46,9 & 5,82 & 17,3 & 90 & 94,66 & 90 & 1 ou $\overline{1}-$ \\
\hline glagolevita & 5,358 & 9,281 & 14,574 & 90 & 97,08 & 90 & 1 \\
\hline lengenbachita & 36,892 & 3,895 & 6,378 & 90 & 90 & 91,01 & 1 ou $\overline{1}-$ \\
\hline pennantita & 5,45 & 9,50 & 14,40 & 90 & 97,3 & 90 & 1 ou $\overline{1}-$ \\
\hline tochilinita & 5,37 & 15,65 & 10,72 & 90 & 95 & 90 & 1 \\
\hline
\end{tabular}


monoclínico só um dos ângulos é diferente de $90^{\circ}$. Pertenceriam, de acordo com sua cruz axial, ao sistema ortorrômbico, mas se enquadram formalmente no sistema monoclínico, pois todos pertencem às classes $2, m$ e $2 / m$.

É preciso então fazer uma série de generalizações. Para as "constantes lineares", se $a\left(a_{1}, a_{2}, a_{3}\right), b$ e $c$ forem tratados como eixos cristalográficos: estará correto dizer que o sinal de igual significa "simetricamente equivalente" e o sinal de diferente significa "simetricamente não-equivalente". Se as mesmas constantes forem pensadas como dimensões da cela unitária: o significado do sinal de igual estará correto e o de diferente deverá significar "geralmente diferente", pois os valores podem ser iguais, apenas por coincidência. Para as constantes angulares, nos dois casos o significado do sinal de igual estará correto e o de diferente deverá significar "geralmente diferente". 1 - SISTEMA TRICLÍNICO: $a, b$ e $c$ são não equivalentes entre si. Normalmente, os ângulos $\alpha, \beta$ e $\gamma$ $\neq 90^{\circ}$, mas poderão ser iguais a $90^{\circ}$, por coincidência. Vemos então que o termo triclínico deixará de fazer sentido! As dimensões dos parâmetros da cela unitária $a, b$ e $c$ serão normalmente diferentes entre si, mas também podem ocorrer coincidências. Caso um dos ângulos seja igual a $90^{\circ} \mathrm{e}$ as dimensões $a, b \mathrm{e}$ $c$ sejam diferentes, ou tenham alguma equivalência por coincidência, o cristal será pseudo-diclínico ${ }^{\star}$ (Tabela 4). Caso dois dos ângulos sejam iguais a $90^{\circ}$ $\mathrm{e}$ as dimensões $a, b$ e $c$ sejam diferentes, ou tenham alguma equivalência por coincidência, o cristal será

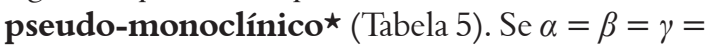
$90^{\circ}$ e as dimensões $a, b$ e $c$ forem diferentes, o cristal será pseudo-ortorrômbico. Se $\alpha=\beta=\gamma=90^{\circ} \mathrm{e}$ duas dimensões forem iguais, o cristal será pseudo-tetragonal. E num caso em que $\alpha=\beta=\gamma=90^{\circ}$ e as dimensões de $a, b$ e $c$ forem iguais, o cristal será pseudo-cúbico. Na prática, o termo "pseudo" não é usado apenas quando os ângulos são exatamente $90^{\circ}$, mas também quando são muito próximos a $90^{\circ}$. *Nota-se que nos casos de cristais pseudo-diclínico e pseudo-monoclínico é o ângulo que necessariamente precisa cumprir as exigências especificadas, podendo os parâmetros ser diferentes ou não (apenas por coincidência). Apesar de nenhum exemplo mineralógico até o momento apresentar essas coincidências quanto à equivalência entre os parâmetros, nada impede que ela possa ocorrer.

2 - SISTEMA MONOCLÍNICO: $a, b$ e $c$ são não equivalentes entre si. Os ângulos $\alpha=\gamma=90^{\circ}$. $\beta$ $\neq 90^{\circ}$, mas poderá ser igual, por coincidência. As dimensões dos parâmetros da cela unitária $a, b$ e $c$ serão normalmente diferentes entre si, mas também podem ocorrer coincidências. Caso $\beta=90^{\circ} \mathrm{e}$ as dimensões $a, b$ e $c$ sejam diferentes, o cristal será pseudo-ortorrômbico (Tabela 6). Se, além de $\beta=$ $90^{\circ}$, duas dimensões da cela forem iguais, o cristal será pseudo-tetragonal. E num hipotético caso em que $\beta=90^{\circ}$ e as dimensões de $a, b$ e $c$ forem iguais, o cristal será pseudo-cúbico.

3 - SISTEMA ORTORRÔMBICO: a cruz axial é formada por 3 eixos não-equivalentes, $a, b$ e $c$. Os ângulos $\alpha=\beta=\gamma=90^{\circ}$, obrigatoriamente. $a, b$ e $c$, sendo não-equivalentes, normalmente exibirão dimensões diferentes. Entretanto, por coincidência, duas dimensões poderão ser iguais, levando a cristais pseudo-tetragonais. Se as três dimensões, por coincidência, forem iguais, os cristais serão pseudo-cúbicos.

4-SISTEMA TETRAGONAL: $a \mathrm{e} b$ são equivalentes entre si e $c$ é diferente $\left(a_{1}=a_{2} \neq c\right)$ e os ângulos $\alpha=$ $\beta=\gamma=90^{\circ}$, obrigatoriamente. Conseqüentemente, as dimensões de $a$ e $b$ (os parâmetros da cela unitária) serão obrigatoriamente iguais. $c$ normalmente terá dimensão diferente, mas poderá, por coincidência, ser de mesma dimensão que $a$ e $b$, apesar

Tabela 6. Parâmetros de cela unitária e classe cristalina de alguns minerais monoclínicos pseudo-ortorrômbicos.

\begin{tabular}{l|l|l|l|l|l|l|l}
\hline mineral & $a(\AA)$ & $b(\AA)$ & $c(\AA)$ & $\alpha\left(^{\circ}\right)$ & $\beta\left(^{\circ}\right)$ & $\gamma\left(^{\circ}\right)$ & classe \\
\hline andorita IV & 13,04 & 17,07 & 10,18 & 90 & 90 & 90 & 2 \\
\hline berilonita & 8,178 & 7,818 & 14,114 & 90 & 90 & 90 & $2 / m$ \\
\hline bijvoetita-(Y) & 21,234 & 12,958 & 44,911 & 90 & 90 & 90 & 2 \\
\hline cymrita & 5,32 & 36,6 & 7,66 & 90 & 90 & 90 & 2 \\
\hline esperita & 17,628 & 8,27 & 30,52 & 90 & 90 & 90 & $2 / m$ \\
\hline ferrierita-Na & 18,886 & 14,182 & 7,470 & 90 & 90 & 90 & $2 / m$ \\
\hline gladiusita & 16,950 & 11,650 & 6,2660 & 90 & 90 & 90 & $2 / m$ \\
\hline gudmundita & 10,00 & 5,95 & 6,73 & 90 & 90 & 90 & $2 / m$ \\
\hline langita & 7,118 & 6,031 & 11,209 & 90 & 90 & 90 & $m$ \\
\hline liveingita & 7,94 & 70,84 & 8,33 & 90 & 90 & 90 & 2 \\
\hline nontronita & 5,23 & 9,11 & 15,5 & 90 & 90 & 90 & $2 / m$ \\
\hline pearceíta-M2a2b2c & 26,036 & 15,0319 & 24,042 & 90 & 90 & 90 & $2 / m$ \\
\hline sarcopsídio & 10,437 & 4,768 & 6,026 & 90 & 90 & 90 & $2 / m$ \\
\hline sartorita & 19,62 & 7,89 & 4,19 & 90 & 90 & 90 & $2 / m$ \\
\hline trimerita & 8,09 & 7,61 & 14,06 & 90 & 90 & 90 & $2 / m$ \\
\hline \hline
\end{tabular}


de não-equivalente (não coincidir com os mesmos elementos de simetria que $a$ e $b$ ). Se a dimensão de $c$ for igual (ou aproximadamente igual) à de $a \mathrm{e} b, \mathrm{o}$ cristal será pseudo-cúbico.

5 - SISTEMA CÚBICO: os eixos cristalográficos $a, b$ e $c$ são obrigatoriamente equivalentes entre si quanto aos elementos de simetria que coincidem com eles, os parâmetros da cela unitária são iguais $\left(a_{1}=a_{2}=a_{3}\right)$ e os ângulos $\alpha=\beta=\gamma=90^{\circ}$, também (obrigatoriamente).

Assim sendo, o que é fundamental para a definição do sistema cristalino é o conjunto de elementos de simetria presentes e não os parâmetros da cela unitária ou a relação angular. Estas são então algumas das convenções inadequadas. Parece lógico pensar que os cristais triclínicos são aqueles que têm todos os ângulos diferentes de $90^{\circ}$. E que os cristais monoclínicos são aqueles que apresentam apenas um ângulo diferente de $90^{\circ}$. Por esse mesmo princípio deveria existir o sistema "diclínico", não? ou seja, um sistema formado por cristais com dois ângulos iguais a $90^{\circ}$ e um diferente! No entanto, os cristais diclínicos se encontram agrupados no sistema triclínico, e a explicação é muito simples: os cristais "diclínicos" apresentam as mesmas classes cristalinas que os cristais triclínicos. Logo, não há sentido em criar um sistema diclínico, mas também não há sentido em utilizar tal nomenclatura confusa.

As convenções para nomenclatura de sistemas cristalinos poderiam ser então diferentes, ou seja, mais lógicas. Os nomes dos sistemas tetragonal (que têm um eixo 4 ou $\overline{4}$ ), trigonal (que têm um eixo 3 ou $\overline{3}$ ) e hexagonal (que têm um eixo 6 ou $\overline{6})$ parecem não ter problemas, pois seu nome é ligado diretamente aos elementos de simetria que os caracterizam. Os demais nomes (cúbico, ortorrômbico, monoclínico e triclínico) são ligados às celas unitárias. Uma opção seria utilizar para estes sistemas os nomes sugeridos por Peacock (1950), que se relacionam aos elementos de simetria. O sistema cúbico (que tem quatro eixos 3 ou $\overline{3}$ ) passaria a se denominar tetra-trigonal, o sistema ortorrômbico (que apresenta três eixos 2 ou $\overline{2}(=\mathrm{m})$ ) passaria a se chamar tri-digonal, o monoclínico (que só apresenta $\underline{\mathrm{um}}$ eixo 2 ou $\overline{2}(=\mathrm{m})$ ) seria nomeado sistema digonal e o sistema triclínico (que só apresenta eixo 1 ou $\overline{1}(=\mathrm{i})$ ) poderia ser renomeado sistema monogonal. Estes quatro nomes são lógicos e tecnicamente adequados, ao contrário dos que são usados oficialmente, mas certamente haveria resistência para substituir termos tão enraizados, como já aconteceu em 1950.

\section{Referências}

Bloss F.D. 1971. Crystallography and crystal chemistry: an introduction. Holt, Rinehart and Winston, 545 p.

Chvátal M. 2007. Mineralogia para principiantes: Cristalografia. São Paulo: Soc. Bras. Geologia. 232p.

Klein C., Dutrow B. 2012. Manual de ciências dos minerais. 23ed., Bookman. 716p.

Peacock M.A. 1950 Remarks on crystallographic nomenclature. Am. Mineralogist 35:882-888.

Pertlik F. 2006. Argumente für die Existenz eines diklinen Kristallsystems in der Fachliteratur des 19. Jahrhunderts. Ein Beitrag zur Geschichte der Kristallographie. - Mitt. der Österreichischen Mineralogischen Gesellschaft 152:17-29.

Roger A.F. 1939. Merosymmetry versus merohedrism. Mineralogical Magazine, 25:252-258.

\begin{abstract}
Resumo: 0s livros de introdução à cristalografia apresentam os sistemas cristalinos de acordo com o conjunto de elementos de simetria e as orientações axiais dos cristais. Entretanto, 0 que é fundamental para a definição do sistema cristalino é apenas 0 conjunto dos elementos de simetria e não as orientações axiais. Alguns dos sistemas cristalinos possuem seus nomes relacionados aos elementos de simetria, como é 0 caso dos sistemas trigonal, tetragonal e hexagonal. Os outros sistemas cristalinos, no entanto, têm seus nomes relacionados à orientação axial: cúbico ou isométrico, ortorrômbico, monoclínico e triclínico. Parece lógico pensar que os cristais do sistema triclínico são aqueles que possuem todos os ângulos diferentes de $90^{\circ}$ e que os cristais do sistema monoclínico possuem dois ângulos iguais a $90^{\circ}$ e apenas um ângulo diferente de $90^{\circ}, 0$ que não é sempre 0 que ocorre. Cristais "diclínicos", com dois ângulos diferentes de $90^{\circ} \mathrm{e}$ um ângulo igual a $90^{\circ}$ também existem! Mas são agrupados no sistema triclínico, pois apresentam grupos pontuais equivalentes. As convenções de nomenclatura dos sistemas cristalinos poderiam ser mais lógicas. Uma opção seria utilizar para todos os sistemas cristalinos a nomenclatura referente aos elementos de simetria. Como resultado 0 sistema cúbico seria denominado sistema tetra-trigonal, o sistema ortorrômbico seria sistema tri-digonal, o sistema monoclínico passaria a ser denominado sistema digonal, e o sistema triclínico seria chamado sistema monogonal. Ao contrário dos nomes que são utilizados oficialmente, esses quatro nomes sugeridos são mais lógicos e tecnicamente corretos.
\end{abstract}

Palavras-chave: sistemas cristalinos, cruz axial, sistema diclínico, nomenclatura cristalográfica, elementos de simetria. 